\title{
Cardiovascular, metabolic and kidney disease: crosscutting science and best practice in multimorbidity - a multispecialty conference at the Royal College of Physicians 鉴
}

\author{
Authors: Elena Cowan, ${ }^{\mathrm{A}}$ Michael Nation, ${ }^{\mathrm{B}}$ Michelle Whittle ${ }^{\mathrm{C}}$ and Phillip A Kalra ${ }^{\mathrm{D}}$
}

Patients with multimorbidity are increasingly encountered, especially with an ageing population and the co-segregation of lifestyle diseases such as diabetes, obesity and hypertension, but the care of these patients is fragmented and research rarely undertaken within this group. Research into genetic biomarkers and the evolution of crosscutting multiorgan science, resulting in collaboration between specialties for the treatment of patients with multimorbidity, should be the next major step change in medicine. Evolving technology is making this possible. However, there is a necessity to instigate more collaborative multispecialty research efforts to provide the evidence needed to move treatment possibilities forward, leading to the capability for a major redesign of clinical practice. The patient must be at the centre of a new, radically changed and holistic journey and collaborative research with primary care is essential, as general practitioners and primary care colleagues are the experts dealing with common multimorbidities, including those due to long-term poor lifestyle.

KEYWORDS: Multimorbidity, cross-specialty collaboration, socioeconomic inequalities

\section{Introduction}

It is well-recognised that along with population ageing, multimorbidity is one of the major burdens to healthcare. Defined as the coexistence of two or more chronic conditions, multimorbidity does not necessarily relate only to older people: nearly one-third of people with three and more chronic conditions are aged less than 65 years. ${ }^{1,2}$

It is also now well established that multimorbidity is associated with worse outcomes: less life expectancy, poor quality of life, as well as higher use of healthcare resources.

Authors: A cardiology research fellow, Queen Alexandra Hospital, Portsmouth, UK; ${ }^{B}$ director of development, Kidney Research UK, Peterborough, UK; ${ }^{C}$ freelance medical writer and proofreader, Lymm, UK; D professor of nephrology, Salford Royal NHS Foundation Trust and University of Manchester, Manchester, UK
The importance of multimorbidity has been acknowledged by the clinical and research communities (the National Institute for Health and Care Excellence (NICE) and the Medical Research Council (MRC)), by charitable organisations (eg Richmond charity groups) and it is at the heart of The NHS Long Term Plan. ${ }^{1-6}$ It was therefore considered timely to develop and host a conference that brought together patients, policy makers, clinicians, researchers, industry partners and funders in order to stimulate discussions about evidence gaps, research needs, underpinning yet underutilised resources and to encourage collaboration in the field. Hence on 01 July 2019 'Cardiovascular, metabolic and kidney disease: crosscutting science and best practice' was held at the Royal College of Physicians (RCP) on behalf of Kidney Research UK (KRUK), the Renal Association (RA), the National Institute of Health Research (NIHR) and the RCP."

The following report encapsulates the key issues that the speakers and delegates agreed need to be addressed to improve the care and future health of the multimorbidity patient, and the potential solutions that were discussed to fulfil this are then summarised.

\section{Current limitations in care and research of multimorbidity patients}

Healthcare systems not suited to the multimorbidity patient

The current healthcare system, which was set up to deal with episodic disease, is now outdated and not geared for efficient treatment of multimorbid patients. Guidelines are specialty specific or based on single disease states and the majority of them do not take into account other comorbidities that patients may have. The fundamental drive to see increased patient numbers means that short appointment lengths do not enable clinicians to undertake comprehensive assessments of complex patients with multiple chronic conditions, patients in whom it is often difficult to ascertain interactions of conditions or of medications used to treat them, and side effects of medications.

Single organ disease approach leading to fragmentation of care and increased polypharmacy

Most medical specialties focus on pathology in the respective single organ thus contributing to fragmentation of care for multimorbid 
patients. These patients attend multiple appointments but still do not receive holistic care. Patients feel that they spend too much time being 'batted' between specialties and see a lack of coordination when navigating the healthcare system. Some feel that they are treated as a disease rather than as a patient, and that their care is being controlled rather than being inclusive of their views. Adherence to different guidelines which do not consider multimorbidity puts patients at risk of polypharmacy. Polypharmacy, in turn, may lead to a prescribing cascade (when new medications are introduced to treat side effects of ongoing medical therapy) with subsequent increased risk of adverse drug reactions and clinical outcomes and a further negative impact on quality of life.

\section{Socioeconomic deprivation as a driver of} multimorbidity and mental-physical disease interplay

Multimorbidity is more common in deprived areas, where it can manifest up to 10-15 years earlier in a patient's life compared with affluent areas. ${ }^{8}$ The prevalence of mental health disorders is increasing in patients with a number of chronic physical conditions. Conversely, those with mental health issues have high risks of developing physical diseases and having difficulties with self-management. Socioeconomic deprivation exacerbates the negative effects of interaction between these mental and physica components. ${ }^{9}$ However, at present there is insufficient recognition of this tandem; mental and physical conditions are viewed by the healthcare system and by clinicians as separate entities.

\section{Current organ-specific data resources}

Most clinical data are currently maintained in organ-centric databases (eg National Institute for Cardiovascular Outcomes Research (NICOR) and UK Renal Registry (UKRR)). There is a general lack of awareness and use of other datasets outside of the single organ-focused interests of the researchers. The same applies to research in our UK clinical cohorts which should be available for more collaborative multispecialty analysis. The potential of the current databases and patient cohorts are far from being fully realised.

\section{Lack of research and evidence of treatment in multimorbid patients}

Historically, patients with multimorbidity have been excluded from clinical research, and hence clinicians are more or less 'in the dark' about managing these patients as evidence is insufficient. There has been an apparent lack of interest in conducting multimorbidity research by the pharmaceutical industry for several reasons. Currently used markers of efficacy are substandard and assessment of standard long-term clinical end-points (eg mortality) can be unrealistic in these heterogeneous patients with competing morbidities or will require a very large number of recruits in extremely expensive randomised controlled trials (RCTs). Where multimorbid patients have been the subject of an RCT, the study end-points have usually concentrated on specialtyspecific outcomes rather than an overall outcome relevant to the many facets of the patients' illnesses. Also, in this group of high-risk patients the outcomes most relevant to the patients themselves, patient-reported outcome measures (PROMs) and patient-reported experience measures (PREMs), have rarely been investigated.

\section{Possible solutions}

\section{Increased awareness of multimorbidity}

Further wider acknowledgement of the multimorbidity epidemic and the challenges it brings for patients and to the healthcare system should be addressed at different levels and incorporated in care systems, in education and training of healthcare workers and bespoke multimorbidity research and guidelines need to be developed.

\section{Further research on multimorbidity}

The lack of evidence and difficulties for the pharmaceutical industry to undertake research on multimorbidity might be addressed by initiating more investigator led trials. There is great opportunity for industry and researchers to have a joint focus on common pathological pathways (eg inflammation or fibrosis) that affect different systems (eg cardiac failure or chronic kidney disease). Adaptive trials with use of appropriate biomarkers to identify more specifically the population at risk (and, at the same time, those who could benefit the most from intervention) would enable more efficient trial design with selection of 'case' groups who can be compared with those who are at no or lower risk of the disease (the 'control' group) thus avoiding high costs of much larger RCTs in heterogeneous populations.

Use of basic science, 'omics' and epidemiological data for better study of multimorbidity

Major benefits could result from wider, non-organ specific analysis of existing single organ / system disease datasets (eg NICOR, UKRR Sentinel Stroke National Audit Programme (SSNAP) and National Diabetes Audit (NDA)) and ongoing cohort studies, the latter enabling both retrospective and prospective multisystem analysis. Better data linkage between datasets and other valuable sources of information at population level (Hospital Episode Statistics (HES) and civil registrations - information on deaths) is another 'low hanging fruit' that would facilitate multimorbidity research.

Knowledge is now emerging on how different long-term conditions tend to coexist or cluster (eg obesity, diabetes and cardiovascular diseases), and this can provide the framework for future research on possible common causes, pathways and the possibilities for prevention. There has been extensive research on biomarkers but their use in adaptive trial design, as mentioned above, has not been realised to any extent. Certain imaging techniques can reduce the need for invasive tests (eg renal magnetic resonance imaging instead of renal biopsy to characterise inflammation, fibrosis and disease progression) with subsequent reduction of complication risks and these being more patient friendly.

The developing world of genomics presents additional opportunities. In diabetic cohorts the use of genetic risk scores has recently assisted traditional clinical methods in distinguishing different subtypes of diabetic patients, crucial as responses to treatments are widely different. Work is currently under way to enable use of genomic information obtained from research in the common diseases implicated in multimorbidity (coronary artery disease, Alzheimer's disease etc) in order to better identify at risk patients and develop clearer understanding of pathogenetic pathways.

There is a need for greater use of artificial intelligence, machine learning and high throughput technologies in the processing, 
integration and making sense of big data, biomarker, genetic and other 'omic' resources.

\section{Integrated care and cross-specialty working}

Healthcare professionals must leave their specialty-specific silos and work across specialties. A better balance between specialist knowledge and 'generalism' is needed. Patients with multimorbidity require a healthcare professional who can coordinate their care. In a limited workforce, the experience and skills of other healthcare professionals should be better utilised (eg pharmacists who will identify the risk of polypharmacy, drug interactions and opportunities for deprescribing; and healthcare navigators to assist patients around the healthcare system).

\section{Addressing inequalities}

Resources should be allocated to where they are needed most - ie in most deprived areas. The major challenge of mental illness in the multimorbid patient is recognised and there should be better communication between physicians and specialists in mental health. Further adoption of e-health technologies should be undertaken with caution so as not to widen the existing inequality gap.

\section{Patient involvement}

Innovative ways of research for multimorbid patients are required - these patients already visit multiple specialists in primary/secondary care and additional visits to a care setting for research purposes may not be attractive or feasible for them. Wider incorporation of PROMs in clinical practice and research will make the patient voice stronger. Dissemination of research results to patients and a simple 'thank you' for taking part in research must become routine parts of the research process.

\section{Conclusion}

We must face reality and accept that multimorbidity is now the norm in our everyday clinical practice. Although as clinicians, we can retreat to our comfort zones of specialist knowledge, patients do not have this option. Multimorbidity patients often originate from or are based in disadvantaged backgrounds and they are likely to be set on a trajectory of poor clinical outcomes and quality of life. It is within the clinicians, researchers and funders remit to alter this trajectory by working together and across specialties, providing integrated care and research for the patient as a whole, rather than dividing care and research into a conglomerate of single pathologies.

This is an exciting time in the evolution of medical practice, and we need to make it happen. Action is required now, and changes in education and training are necessary to ensure that future clinicians are well prepared to embrace the complexities of multimorbidity.

\section{Acknowledgements}

For helping to organise the conference: Emma Woollard, development coordinator for research, Kidney Research UK; Emanuela Mariani, specialty cluster A acting assistant lead and general manager, Clinical Research Network National Coordinating Centre, National Institute for Health Research; and the Royal College of Physicians.

For sponsorship and endorsement: Renal Association; Kidney Research UK; and the National Institute for Health Research.

\section{References}

1 National Institute for Health and Care Excellence. Multimorbidity: clinical assessment and management. NICE guideline [NG56]. London: NICE, 2016. www.nice.org.uk/guidance/ng56.

2 Stafford M, Steventon A, Thorlby R et al. Briefing: Understanding the health care needs of people with multiple health conditions. The Health Foundation, 2018. www.health.org.uk/publications/ understanding-the-health-care-needs-of-people-with-multiplehealth-conditions.

3 Medical Research Council. Delivery plan 2019. MRC, 2019. www. ukri.org/files/about/dps/mrc-dp-2019.

4 The Academy of Medical Sciences. Multimorbidity: a priority for global health research. London: The Academy of Medical Sciences, 2018. https://acmedsci.ac.uk/policy/policy-projects/multimorbidity.

5 Aiden H. Multimorbidity. Understanding the challenge. London: The Richmond Group of Charities, 2018. https://richmondgroupofcharities.org.uk/sites/default/files/multimorbidity___understanding_ the_challenge.pdf

6 NHS. The NHS Long Term Plan. NHS, 2019. www.longtermplan.nhs. uk

7 Royal College of Physicians. Cardiovascular, metabolic and kidney disease: crosscutting science and best practice. London: RCP, 2019. www.rcplondon.ac.uk/events/cardiovascular-metabolic-and-kidneydisease-crosscutting-science-and-best-practice

8 Barnett K, Mercer SW, Norbury M et al. Epidemiology of multimorbidity and implications for health care, research, and medical education: a crosssectional study. Lancet 2012;380:37-43.

9 Naylor C, Parsonage M, McDaid D et al. Long-term conditions and mental health. The cost of co-morbidities. King's Fund and Centre for Mental Health, 2012. www.kingsfund.org.uk/publications/longterm-conditions-and-mental-health

Address for correspondence: Elena Cowan, cardiology research team, Queen Alexandra Hospital, Southwick Hill Road, Cosham PO6 3LY, UK.

Email: cowan.elena@gmail.com 